\title{
Configuración histórica de la discapacidad visual y sus implicaciones para la salud pública
}

\author{
Historical configuration of visual impairment \\ and the implications for public health
}

\author{
María del Pilar Oviedo-Cáceres ${ }^{1}$; Samuel-Andrés Arias-Valencia²; Andrea Hernández-Quirama³
}

Forma de citar: Oviedo Cáceres MP, Arias Valencia SA, Hernández Quirama A. Configuración histórica de la discapacidad visual y sus implicaciones para la salud pública. Rev Univ Ind Santander Salud. 2019; 50(3): $252-261$. doi: http://dx.doi.org/10.18273/revsal.v51n3-2019008 (c) (i)

\begin{abstract}
Resumen
Las personas con discapacidad, han sido objeto de diferentes miradas que dan cuenta de la manera como históricamente han sido significadas y ubicadas en la realidad social. Este trabajo tiene como objetivo hacer una reflexión con relación a la configuración del constructo de discapacidad visual al interior de los modelos teóricos sobre el tema y las implicaciones que han tenido en la manera como se interviene desde la salud pública. El modelo de la diversidad funcional, así como la interseccionalidad se constituyen en elementos clave que deben ser incluidos en la reflexión del quehacer en la salud pública alternativa, pues permiten hacer un ejercicio comprensivo de la realidad de las personas con diversidad funcional visual, y tener herramientas para superar las discriminaciones y establecer las condiciones para la garantía de sus derechos humanos, aspectos fundamentales desde la mirada política y técnica de la salud pública.
\end{abstract}

Palabras clave: Personas con discapacidad; Baja visión; Salud pública.

\begin{abstract}
People with disabilities have been subjected to different looks, which account for how historically they have been significant and placed in social reality. This work aims to reflect on the configuration of the visual disability construct within the theoretical models on the subject and the implications that have had on the way in which it is intervened from public health. The model of functional diversity as well as intersectionality are key elements that must be included in the reflection of the work in alternative public health, as they allow to make a comprehensive exercise of the reality of people with functional diversity and having tools to overcome discrimination and establish the conditions for the guarantee of their human rights, fundamental aspects from the political and technical perspective of public health.
\end{abstract}

Keywords: People with disabilities; Low vision; Public health.

1. Universidad CES. Medellin, Colombia.

2. Universidad de Antioquia. Medellín, Colombia.

3. Universidad Industrial de Santander. Bucaramanga, Colombia.

Correspondencia: María del Pilar Oviedo. Dirección: Calle 10A 22-04. Teléfono: +54 4440555 Ext 1963. Correo electrónico: oviedoc.maria@uces.edu.co 


\section{Introducción}

Las personas con discapacidad, han sido objeto de diferentes miradas que en su gran mayoría dan cuenta de la manera como históricamente han sido significadas y ubicadas en la realidad social de los grupos; una significación que va desde la deficiencia, la caridad, la compasión y la lástima, hasta enfoques que buscan la reivindicación del sujeto, con un énfasis en las capacidades, la diversidad de la vida humana y el reconocimiento de sus derechos ${ }^{1,2}$. Históricamente la discapacidad ha sido analizada desde diferentes aspectos, pasando de una perspectiva relacionada con el castigo divino y la brujería, a enfoques que buscan reivindicar a las personas en su dignidad humana y en sus derechos.

Si bien, se reconoce una evolución de la forma como la sociedad ha venido haciendo la construcción conceptual y social de la discapacidad, se han propuesto enfoques más progresistas, en los cuales el papel activo que desempeñan las personas con discapacidad ha sido fundamental en términos de igualdad y no discriminación; sin embargo, aún persisten miradas limitantes que conviven simultáneamente, lo cual, ha dificultado la superación de esquemas ligados a una historia de beneficencia y caridad ${ }^{3}$.

La manera como se construye el concepto de discapacidad, tiene un efecto directo en la respuesta social, ya que es diferente la forma como se interviene la situación cuando se interpreta desde el principio de la "normalización" o si se hace desde una perspectiva como la de la "diversidad funcional" en la que se parte de la realidad de la diversidad del ser humano, tanto dentro de su propia vida, como de un ser humano a otro, y considera que ésta es una fuente de riqueza ${ }^{4}$.

Desde la normalización, y teniendo en cuenta las formulaciones realizadas por Foucault ${ }^{5}$, se resalta el hecho de que sobre los cuerpos se inscriben normas de regulación de la existencia; se trata de prescripciones normativas en las que lo "normal" cumple la doble función de señalar una regularidad estadística (el caso mayoritario) y una prescripción moral (lo bueno y adecuado; lo deseable), esto obliga a la homogeneidad, anulando todo aquello que se escape de la norma.

En el caso de las personas con discapacidad, esa naturalización del cuerpo hace que las directrices normalizadoras sean estipuladas por la ciencia médica, de modo que se establece una indisociable vinculación entre discapacidad y enfermedad, haciendo que la respuesta social esté ordenada en términos de rehabilitación clínica y funcional, desdibujando al individuo ${ }^{3}$.

Por su parte, desde la diversidad funcional, se reconoce la dignidad intrínseca desde la igualdad del valor de las vidas de todas las personas y extrínseca desde la igualdad de derechos de todos los seres humanos. Esta mirada implica utilizar novedosas aproximaciones que permitan introducir en el corazón del debate bioético, el verdadero sustento de la dignidad intrínseca de las personas que son discriminadas ${ }^{4}$.

De forma tradicional, la discapacidad visual se ha abordado desde una perspectiva biomédica, lo cual se refleja en el constructo que se tiene tanto de la ceguera como de la baja visión, ya que desde este modelo se desconoce e invisibiliza al individuo, sus capacidades, potencialidades y necesidades ${ }^{5}$. En ese sentido, la baja visión, se ha definido como una categoría de deficiencia visual de moderada a grave en la que se presenta una dificultad para percibir luz, forma, tamaño o color de los objetos, en la cual las personas que la padecen experimentan limitaciones para el desarrollo de distintas actividades básicas de la rutina diaria como caminar, salir a la calle, cocinar, realizar pasatiempos, así como el desarrollo de tareas que requieran de la visión, tales como el estudio y el trabajo; trayendo como consecuencia restricciones en la participación del individuo, en el ejercicio de sus derechos, en la producción de ingresos, en la toma de decisiones, en el acceso a los espacios tecnológicos, arquitectónicos, entre otros, generándose así una condición de discapacidad visual ${ }^{6}$. En cuanto a la ceguera, ésta corresponde a aquellas personas con una agudeza visual que va desde 20/400 hasta la No Percepción Luminosa (NPL).

Este artículo tiene como objetivo hacer una reflexión en relación con la configuración del constructo de discapacidad visual al interior de los diferentes modelos teóricos, como el de prescindencia, el médicobiológico o rehabilitador, el social, el biopsicosocial y finalmente el de diversidad funcional y las implicaciones que estos han tenido en la manera como se interviene desde la salud pública. Ofreciendo una contribución al estudio del fenómeno de discapacidad visual para orientar la discusión sobre la necesidad de nuevas herramientas como la interseccionalidad, que permitan trabajar por la garantía de los derechos de las personas con esta condición. 


\section{Modelo de prescindencia}

Este modelo se sitúa en la antigüedad clásica y en las sociedades griegas, se caracterizó por la consideración de la discapacidad como una situación producto de un castigo divino, según el cual, las personas con esta condición eran concebidas como innecesarias puesto que no contribuían a las necesidades de la comunidad ${ }^{1,7}$. Dentro de este modelo se ubican prácticas que involucran, por un lado, el exterminio, la marginación y la segregación, por otro, la caridad, la lástima y el proteccionismo hacia las personas consideradas como "anormales" . Es así como, en este contexto surgieron modelos eugenésicos y de marginación para las personas con discapacidad visual, los cuales se reflejaban en el abandono de los bebés ciegos; aquellos que lograban sobrevivir o las personas mayores recurrían a la caridad y al ejercicio de la mendicidad como estrategias o medios de subsistencia obligados. Con la influencia de la religión, pasó a considerarse a las personas con discapacidad visual como protegidos por los dioses y portadores de virtudes especiales, pues contaban con una iluminación interior que les ponía en relación directa con el mundo invisible ${ }^{9}$.

\section{Modelo médico-biológico o rehabilitador}

Este modelo surge después de la primera guerra mundial y entiende la discapacidad como una anomalía, que no tiene su origen en un castigo divino sino en una imperfección física, psíquica o sensorial, congénita o adquirida, que sitúa a algunos sujetos por debajo de unos niveles considerados como "normales"10. Teniendo en cuenta sus raíces médicas, la discapacidad bajo este modelo se entiende, ya sea como una condición negativa o deficitaria del sujeto que la padece, o un comportamiento desviado y síntoma de una alteración biológica subyacente.

Desde esta perspectiva, la persona con discapacidad se reconoce como un sujeto enfermo, y por tanto necesita ser rehabilitado mediante la intervención de profesionales médicos para lograr su normalidad; en esta medida, la rehabilitación se enfoca en ocultar o desaparecer la diferencia, y el éxito es valorado en relación con la cantidad de destrezas y habilidades que logre adquirir la persona ${ }^{7}$. Como consecuencia, desde las posturas de la medicina clínica es el individuo el que tiene que supeditarse, tanto en el ámbito de la comprensión como en el de su accionar, a los dictámenes del médico, representante del conocimiento y del método científico ${ }^{11}$.
Uno de los componentes más significativos de este modelo es que la persona asume un rol de enfermo e individuo pasivo sin tenerse en cuenta sus derechos ${ }^{12}$. Este modelo se centra en la discapacidad desde el punto de vista de las actividades que la persona no puede realizar, por lo que se produce subestimación con relación a las aptitudes de los sujetos, lo cual ha llevado a producir estigmatización y marginación; mirada que intenta corregir lo que considera el fundamento objetivo de su deficiencia y se refleja en la clasificación internacional de deficiencias, discapacidades y minusvalías (CIDDM) de la Organización Mundial de la Salud, (OMS) la cual incluye el término "handicap" como minusvalía, dando así un significado negativo o peyorativo, ya que minusvalora a la persona y la sitúa en un grado inferior a las demás ${ }^{13}$. Este acercamiento en relación a la falta de capacidades, se refleja en algunas conceptualizaciones de baja visión en las que se define la condición como "una pérdida irreversible de la visión que impide (seriamente) la capacidad de un individuo para aprender o realizar alguna o todas las tareas habituales y adecuadas a su edad, aunque aún permite algún uso funcional de la visión para las actividades diarias" ${ }^{\prime 1}$.

Otro aspecto importante a mencionar, tiene que ver con la mirada capitalista al asunto de la rehabilitación, pues ésta se ve como una posibilidad de que los individuos puedan aportar a la comunidad, considerarse rentables y adaptarse a las demandas del entorno una vez logren la normalización de sus funciones, de tal manera que es la persona con discapacidad la que tiene dificultades para realizar actividades, lo que le obstaculiza desempeñar un rol social de acuerdo con las expectativas normativas del medio ${ }^{1}$. En este sentido, las definiciones de discapacidad desde el modelo médicobiológico se enfocan en la deficiencia como el factor que determina la condición de discapacidad, al subrayar las restricciones o pérdidas presentes en la persona que afectan su capacidad para realizar una actividad en la forma o dentro del margen considerado como normal ${ }^{15}$.

La mirada orgánica que reconoce la discapacidad visual en el cuerpo, permanece arraigada en escenarios académicos, normativos y sociales, prueba de esto es la denominación que se daba a la condición, según la cual, una persona que no tenga los estándares visuales establecidos se clasificaba bajo un diagnóstico de "visión subnormal" el cual fue incluido en la Clasificación Internacional de las Enfermedades CIE10 de la Organización Mundial de la Salud (OMS) desde 1990, aunque después, este término y el de baja visión fueron removidos de la clasificación en el año 
2009 y reemplazados por discapacidad visual moderada y severa ${ }^{16,17}$. En el contexto de este modelo, la atención sanitaria se considera primordial y en el ámbito político, la respuesta principal es la de modificar y reformar la política de atención a la salud, limitando el accionar de las mismas a aspectos preventivos con el objeto de evitar las causas y/o disminuir las consecuencias de la discapacidad, privilegiando la rehabilitación, pues lo que se busca es ajustar a la persona a parámetros sociales a través del manejo de un profesional que trata, cambia y "mejora" la persona como objeto" ${ }^{18}$. Para el caso específico de las personas con discapacidad visual en el marco de este modelo, la rehabilitación tiene como objetivo dotar a las personas de todo tipo de técnicas, estrategias y recursos que les permitan realizar las actividades cotidianas, participando de forma activa en cualquier entorno (educativo, laboral, cultural, de ocio, etcétera) para conseguir una integración social normalizada; dicha rehabilitación varía en general en el tiempo de entrenamiento con cada paciente, de acuerdo con el tipo de ayudas ópticas que necesiten para cada actividad y a la visión residual funcional que tengan ${ }^{19}$.

\section{Modelo social}

Este modelo surge los años 60, como consecuencia de las luchas por la vida independiente y ciudadanía o derechos civiles para las personas con discapacidad; de manera tal que deja de ser entendida como una anormalidad de la persona y comienza a ser contemplada como una anormalidad de la sociedad. En este sentido, la discapacidad es producto, de la manera en la que se han construido el entorno, los productos y los servicios e incluso, de la manera en la que se ha concebido al propio ser humano. Bajo esta mirada, las causas que la originan no obedecen a asuntos religiosos o clínicos, sino más bien situaciones sociales, reconociendo que los sujetos pueden aportar a las necesidades de la comunidad en igual medida que el resto, pero siempre desde la valoración y el respeto de su condición de personas diferentes ${ }^{1}$.

En este modelo se defiende que la concepción de la discapacidad es una 'construcción social' impuesta, y plantea una visión de las personas con discapacidad como clase oprimida, con una severa crítica al rol desempeñado por los profesionales de la salud y la defensa de una alternativa de carácter político más que científico ${ }^{20}$. Robert Scott en una obra realizada desde la antropología, señala que la experiencia de la ceguera es construida y aprendida culturalmente, y que la profesión médica posee un papel nodal en la imputación y reproducción de la dependencia ${ }^{21}$. Los principios de este modelo permearon la definición de la discapacidad promovida en la convención sobre los derechos de las personas con discapacidad (2006) de la Organización de las Naciones Unidas, como "Un concepto que evoluciona y que resulta de la interacción entre las personas con deficiencias y las barreras debidas a la actitud y al entorno, que evitan su participación plena y efectiva en la sociedad, en igualdad de condiciones con las demás", constituyéndose en el primer documento internacional que supone un cambio de paradigma en las actitudes y enfoques respecto de las personas con discapacidad ${ }^{1}$.

Los argumentos de este modelo, proponen que la discapacidad nace de la interacción de una persona con un medio ambiente particular, en el cual se evidencian las limitaciones o desventajas que definen su estatus. Bajo esta perspectiva, es el medio social el que no ha tenido la capacidad de dar respuestas a las necesidades de la persona; motivo por el cual se plantea la importancia que tiene la sociedad de organizarse para eliminar las barreras físicas y actitudinales, de manera que el efecto de las deficiencias no vaya más allá del ámbito fisiológico de la persona con discapacidad ${ }^{15}$. Así mismo se encuentra íntimamente relacionado con los derechos humanos y aspira a potenciar el respeto por la dignidad, la igualdad y la libertad, propiciando la accesibilidad universal y proponiendo una alternativa de carácter más político que científico, defendiendo el derecho de las personas con discapacidad de convertirse en sujetos sociales y económicamente activos, cuya opinión debe ser tenida en cuenta a la hora de elaborar políticas de inclusión. Es importante mencionar que ha tenido mayores desarrollos en las personas con discapacidad física y auditiva, sin embargo, en el ámbito de la discapacidad visual, ha sido poco explorado ${ }^{8}$.

\section{Modelo bio-psicosocial}

Surge en 1977, e intenta una lograr una integración coherente de los componentes del modelo médico y del modelo social desde una perspectiva biológica, individual y social, basada en la interacción de una persona con discapacidad y su medio ambiente ${ }^{22}$. Un aspecto diferenciador tiene que ver con que la discapacidad no es vista como una característica de algunos grupos sociales, sino que se trata de una experiencia humana universal, un concepto dinámico bidireccional fruto de la interacción entre el estado de salud y los factores contextuales ${ }^{23}$. Las críticas conceptuales y prácticas al modelo médico, cuya traducción fue la CIDDM de 1980, movieron a la OMS a revisar su clasificación y su definición de discapacidad 
con el propósito de armonizar la aproximación médica y la aproximación social. El resultado de esta síntesis es la Clasificación internacional del funcionamiento, la discapacidad y la salud (CIF 2001).

El cambio de enfoque es perceptible: desde una clasificación de consecuencias de enfermedades (CIDDM 1980) a una clasificación de "componentes de salud" (CIF 2001) de aplicación universal, válida para todas las personas y no sólo para las personas con discapacidad, e integrando aspectos biomédicos y sociales $^{24}$. Las implicaciones de esta concepción pasan por la utilización de un lenguaje neutro y positivo al momento de definir y clasificar la discapacidad. Así, bajo este modelo, la discapacidad no es atributo de la persona, sino un complejo conjunto de condiciones, muchas de las cuales son creadas por el contexto/ entorno social; por lo tanto el manejo del fenómeno requiere la actuación social, y es responsabilidad colectiva de la sociedad hacer las modificaciones ambientales necesarias para la participación plena de las personas con discapacidades en todas las áreas de la vida social ${ }^{25}$. En consecuencia, la discapacidad deja de ser una categoría meramente negativa, pues presenta rasgos que obligan a considerar a la persona a partir de sus capacidades y su actuación o funcionamiento, a la mejora e incremento de tales posibilidades de actuación y a la adquisición de otras mediante adecuados sistemas de apoyo.

Como modelo holístico de salud y enfermedad, considera al individuo un ser central que participa de las esferas biológicas, psicológicas y sociales, pues cada sistema influye en los otros y a su vez está influido por los demás. Se enfatiza en la importancia de la expresión y la participación plena en todos los contextos ambientales capaces de influir positiva o negativamente sobre la persona con discapacidad y, por tanto, su manejo requiere de la participación de otros $^{12}$. En este sentido, toda intervención bajo este modelo, debe estar enfocada en el abordaje de las tres esferas que le dan su denominación para garantizar con éxito una recuperación o un mantenimiento completo de la deficiencia, trastorno o enfermedad ${ }^{25}$. Esta clasificación, aporta un lenguaje común, fiable, estandarizado y aplicable transculturalmente, que permite describir el funcionamiento humano y la discapacidad, utilizando una visión universal de la discapacidad ${ }^{26}$. En sincronía con esta posición, la Asociación Americana de Optometría, realiza un acercamiento al hablar de discapacidad visual como una deficiencia en el funcionamiento de los ojos o del sistema visual que limita la independencia personal o socioeconómica, dado que la condición interfiere de forma significativa en las habilidades que otorgan independencia a persona, entre ellas las que le permiten realizar actividades de la vida diaria o su desplazamiento con seguridad ${ }^{27}$.

\section{El modelo de la diversidad}

Este modelo surge en el año 2006 en España, al interior del colectivo de personas con discapacidad y su derecho a decidir quiénes son por sí mismos, a no ser lo que el calificativo que se les atribuye pretende denotar: discapacitados, personas sin capacidad o capacidades ${ }^{28}$. Insiste en la discapacidad como rasgo que configura decisivamente la identidad personal y proporciona un sentido de pertenencia; la valora de forma positiva como circunstancia que enriquece la identidad de nuestras sociedades: Por tanto, esta condición no impide vivir con plenitud y satisfacción pues se trata únicamente de vivir de una manera diversa ${ }^{24}$.

Este modelo, parte de una postura basada en los Derechos Humanos y propone a su vez un imprescindible cambio terminológico que modifica los términos vinculados a la valía o la capacidad tales como minusválido o discapacidad, y defiende el uso de nuevas expresiones: mujeres y hombres discriminados por su diversidad funcional o, más corto, personas con diversidad funcional ${ }^{4}$. Lo anterior, no implica un simple cambio de nombre, sino un proceso de resemantización activista, de crítica, denuncia y deconstrucción de lo que crean las actuales palabras, sustituyéndolas por otras que digan nuevas cosas para generar así nuevas representaciones y relaciones ${ }^{29}$.

Desde este modelo se indica el hecho de que las personas con diversidad funcional tienen un desenvolvimiento cotidiano diferente al que se considera usual; no obstante, más allá de la denotación, también incorpora el sentido de que esa funcionalidad diversa implica discriminación y que no es la peculiaridad fisiológica, sino el entorno social el que la produce; por tanto, las personas no tienen deficiencia, sino diversidad, de lo cual se deduce que lo que sufren no es discapacidad, sino marginación e injusticia debido a su diversidad. La clave ya no es la capacidad, sino la dignidad del ser humano de tipo extrínseca (relacionada con los derechos y las condiciones de vida de las personas) e intrínseca (relacionada con el valor de la vida de las mujeres y los hombres) y no se habla de normalidad sino de diversidad, la cual se refleja en la propia sociedad actual, diversidad funcional, cultural, religiosa, sexual, de género, étnica, de edad ${ }^{28}$. 


\section{Implicaciones de los modelos de discapacidad desde la salud pública}

Abordar el fenómeno de la discapacidad visual desde las posturas anteriormente expuestas tiene implicaciones en el marco de la salud pública, pues, así como la discapacidad ha sido concebida de forma distinta a lo largo de la historia, la salud pública también ha tenido unas dinámicas y corrientes específicas en las que se identifican la tradicional y la alternativa, la cual responde a posturas críticas con aportes latinoamericanos. La salud pública tradicional en términos de Granda ${ }^{15}$ es considerada como la enfermología pública, pues parte del presupuesto filosófico-teórico de la enfermedad y la muerte para explicar la salud; el método positivista para explicar el riesgo de enfermar, el estructuralfuncionalismo para comprender la realidad social y finalmente el poder del Estado para asegurar la prevención de la enfermedad. Bajo esta mirada, la salud pública permanece primordialmente en el campo de la enfermedad sin trascender hacia el de la salud, se aborda la temática desde un enfoque rehabilitador dado que se entiende la discapacidad como un asunto que está en el cuerpo, que es asumido como enfermedad, por lo cual se configura como un diagnóstico médico y una desviación individual de la normalidad en el funcionamiento humano.

En esta vía, se reconocen los aportes clínicos para el manejo de las implicaciones de la discapacidad visual, y la utilización de la visión residual, que permiten a la persona ajustar y desarrollar habilidades compensatorias para mejorar su funcionalidad, tales como la prescripción de dispositivos técnicos de apoyo como lupas, telescopios, entre otros, que contribuyen a disminuir el impacto negativo de esta condición en el desarrollo de funciones relacionadas como la lectura, la movilidad, el acceso a la información entre otras ${ }^{30,31}$. Sin embargo, esta concepción eminentemente biológica, permite dilucidar la razón por la cual la salud pública tradicional se ha quedado limitada para dar respuesta a problemas de salud propios de la realidad cambiante y compleja de las personas con discapacidad visual y de sus situaciones cotidianas, pues se ha abordado desde la perspectiva higienista, desde el discurso biomédico y de la epidemiología clásica, centrándose por tanto en la enfermedad y su rehabilitación ${ }^{32}$.

En la salud pública tradicional, la respuesta a la discapacidad visual, ha sido la formulación de políticas y planes destinados a mejorar el acceso a servicios de atención ocular en los niveles primarios para la detección y en niveles de complejidad mayor para cirugía y rehabilitación, así como la generación de programas de prevención y educación con un enfoque individual ${ }^{33}$. Desde este enfoque de la salud pública, el acento se encuentra en el papel del Estado y en la prestación de servicios de atención médica mediante un modelo de mercados, el cual para el contexto de las personas con discapacidad visual es un escenario que genera serias implicaciones; un aspecto crítico tiene que ver con el mecanismo de vinculación, pues en muchas oportunidades, no pueden aportar en el marco de los sistemas de salud basados en aseguramiento y por tanto por capacidad de pago, por no estar vinculados a ninguna actividad productiva, pero tampoco pueden acceder programas sociales, ya que viven y dependen de familias que, en algunos casos, no son consideradas en la categoría de pobreza extrema.

Entender la discapacidad visual desde una perspectiva eminentemente biomédica es claramente limitante y desdibuja no solamente al sujeto sino a los aspectos contextuales y sociales que tienen una relevancia significativa en la consecución de una vida digna; pues sin duda ésta es más contextual que individual, lo que demanda acciones orientadas más hacia la inclusión social y no solamente hacia la atención clínica ${ }^{34}$.

En este sentido, es importante hacer una reflexión en torno a la tendencia existente en Colombia, en relación a la implementación de modelos de intervención en discapacidad visual enfocados desde un modelo biomédico y en algunos contextos desde la caridad o filantropía, los cuales no son suficientes para la transformación y mejoramiento de la vida de las personas $^{35}$. Es necesario dar un giro al abordaje desde una perspectiva de la salud pública alternativa, en la cual, retomando a Granda se hace necesaria la reflexión alrededor de la salud y no solo sobre la enfermedad, acercándose a la metáfora del "poder de la vida", lo que implica comprender la salud pública desde la vida misma y no desde el cálculo del riesgo que ocurre por fuera y antes de que la "máquina corporal" enferme, esto implica visitar propuestas teóricas y metodológicas diversas de las recomendadas por la epidemiología, por tanto, se invita a escuchar con más atención las voces de los movimientos sociales para reconstruir la salud pública con mayor pertinencia; al hacerlo, se podría por tanto conformar una respuesta un tanto diferente ${ }^{36}$.

Por tanto, los postulados propuestos por el modelo de diversidad funcional se hacen pertinentes en este contexto, en el cual se reivindique el lugar del sujeto, acercándose a sus circunstancias particulares de vida, haciendo así una reflexión en la cual la discapacidad 
deje de ser un diagnóstico para convertirse en una categoría social que exige ser analizada desde otras orillas, desde los derechos humanos, reconociendo a la persona en su dignidad humana, concibiéndola en su multidimensionalidad. En este sentido se retoma la postura de Franco quien hace un llamado a romper la disciplinariedad médica en la que ha estado amarrada la salud pública, para dejar de lado la construcción de saberes eminentemente paraclínicos y por el contrario se puedan configurar prácticas que den respuesta a las necesidades integrales que en salud demanda la población ${ }^{18}$.

\section{Interseccionalidad como una herramienta para abordar el fenómeno desde la salud pública alternativa}

La interseccionalidad de género surge como una postura de las corrientes de la teoría crítica, la cual propone formas generales de vida más humanas, que luchan porque las diferencias no se transformen en inequidades. Desde la perspectiva de Granda, los movimientos generados a partir de las corrientes feministas, buscan construir sujetos que puedan integrar en su vida su "yo" con todo su recuerdo cultural, pero también puedan ver al otro, construir un nosotros y luchar contra la opresión ${ }^{36}$. Este enfoque en el marco de la diversidad funcional, permite hacer una crítica a la forma como se han enfrentado tradicionalmente los problemas de salud-enfermedadatención, reconociendo las diferencias entre el hombre y la mujer.

La interseccionalidad es una perspectiva teóricometodológica que busca identificar de qué manera la intersección de las estructuras sociales (género, sexualidad, raza, nacionalidad, clase y discapacidad) generan situaciones de discriminación complejas que se mantienen y reproducen tanto en el nivel estructural, como político y discursivo ${ }^{37}$. Por tanto, la interseccionalidad quiere decir que varias formas de discriminación relacionadas con distintas capas de identidad pueden confluir para crear nuevas formas de discriminación, que son únicas. Este concepto fue acuñado en 1989 por la abogada afroestadounidense Kimberlé Crenshaw en el marco de la discusión de un caso concreto legal, con el objetivo de hacer evidente la invisibilidad jurídica de las múltiples dimensiones de opresión experimentadas por las trabajadoras negras de la compañía estadounidense General Motors ${ }^{38}$. Si bien tiene surgimiento en el contexto jurídico, gracias al trabajo de la socióloga Patricia Hill Collins, el concepto de ha traspasado las fronteras disciplinares del derecho llegando a la sociología y a todas las disciplinas de las ciencias humanas y sociales, por lo cual se considera como una de las contribuciones conceptuales más importantes de la teoría feminista en los últimos 25 años. La interseccionalidad se puede definir como una perspectiva que se centra en las desigualdades sociales y analiza las interacciones entre estructuras sociales, representaciones simbólicas y procesos de construcción de la identidad que son específicos de cada contexto e inextricablemente vinculados a la praxis política ${ }^{39}$.

Uno de los principales alcances de este enfoque es que se aleja de cualquier tipo de generalización y propone un nuevo orden de complejidad para entender de qué manera el sexo y el género se interrelacionan con otras dimensiones de inequidad social, en contextos históricos y geográficos específicos, de los cuales los individuos forman parte para crear experiencias únicas en las áreas de salud. En ese sentido, hablar de interseccionalidad no se reduce a la suma de condiciones de vulnerabilidad sino de la relación dinámica entre los determinantes sociales, el sujeto y su contexto histórico y cultura ${ }^{40}$. Por tanto, emerge como un camino coherente para la comprensión de los procesos de salud-enfermerdadatención, además permite reconocer que las experiencias interseccionales de discriminación están asociadas al estado de salud de la población.

La interseccionalidad ha sido fundamental en la medida en que ha servido para visibilizar violaciones de derechos humanos que antes no se detectaban porque se analizaba la discriminación solamente desde una perspectiva como, por ejemplo, la etnicidad, el color de la piel, el género, la diversidad funcional, la orientación sexual o la edad. Por tanto, permite mostrar la interconexión y contextualidad de las relaciones de poder que configuran la estructura social, permite abogar por la justicia social como parte de un esfuerzo más amplio que incluye la crítica al status quo y la mejora de las condiciones de vida de las personas más marginadas y oprimidas ${ }^{39}$.

Desde la salud pública alternativa y retomando a Granda, se hace necesario escuchar con más atención las voces de los movimientos sociales y en este caso de las corrientes surgidas por los movimientos feministas y su enlace con la diversidad funcional, para reconstruir una salud pública con mayor pertinencia; lo cual permitiría conformar una respuesta diferente a la que tradicionalmente se ha dado, acercándose así un poco a la metáfora del "poder de la vida" ${ }^{36}$. El llamado es a actuar sobre la salud y la vida de las personas con diversidad funcional visual, y no solamente sobre la deficiencia biológica ocular, a entender la diversidad 
como producción social, enmarcada en una realidad histórica y sociocultural concreta, lo que implica que el centro del quehacer deje de ser la intervención sobre las funciones corporales, sino el sujeto en su entramado histórico y en su contexto, teniendo como presupuesto filosófico-teórico la salud y la vida, utilizando métodos variados que permitan ser capaces de dar cuenta de la acción social y de las estructuras; concebiendo al ser humano como ser holístico e integral. El punto de encuentro de la interseccionalidad y la salud pública está entre otros, en el entrecruzamiento de los diferentes ejes de discriminación y su impacto, traducidos en inequidades en salud que viven las personas con diversidad funcional visual, por tanto la salud pública debe generar una postura crítica sobre cómo los sujetos viven, para aportar a su transformación y reducir las inequidades en salud, siendo la mirada interseccional pertinente para dicho $\mathrm{fin}^{41}$.

\section{Conclusiones}

La discapacidad ha sido históricamente abordadas desde un enfoque biomédico-rehabilitador, lo cual, ha tenido consecuencias muy importantes en la percepción actual de la sociedad sobre las mujeres y hombres con esta condición. El impacto más importante viene de la taxonomía basada en la percepción médica de la misma, es decir, en función de la patología o la diferencia funcional de un individuo $\operatorname{concreto}^{42}$. Pese a lo anterior, se constituyen en categorías sociales, situadas históricamente que requieren de una reflexión que se distancie de estos abordajes y es en este contexto que el modelo de la diversidad funcional y sus principios de reivindicación de la dignidad humana y de los derechos humanos se constituyen en elementos claves que deben ser incluidos en la reflexión del quehacer en la salud pública alternativa, pues hablar desde la orilla de la diversidad funcional, permite dejar de considerar la persona como objeto de medidas asistenciales, de beneficencia o caridad y reconocerles como sujeto de derechos, abriendo caminos y dando luces para la búsqueda de la equidad y horizontes orientados al bienestar de este colectivo ${ }^{3}$.

En este sentido, el enfoque de la interseccionalidad ${ }^{43}$, permite un acercamiento a la experiencia de las personas con diversidad funcional, dando elementos para la comprensión de las marginaciones que viven, y permite romper con los paradigmas hegemónicos de normalización del cuerpo o del saber, y hacer visibles las opresiones, las relaciones de poder y de dominación que han existido históricamente, lo cual hace posible no solo develar las desigualdades y discriminaciones sino a su vez generar saberes situados que reconocen al individuo y sus experiencias únicas que resultan de la conjunción de diferentes tipos de identidad ${ }^{44}$. Por tanto el enfoque de la interseccionalidad, permite la reivindicación del sujeto gracias al acercamiento a la comprensión desde una mirada de "abajo hacia arriba", dando cuenta de los distintos elementos que influyen en la vida de las personas con diversidad funcional visual y comprendiendo cómo determinadas políticas y prácticas configuran sus vidas. Lo anterior, demanda un nivel de mayor complejidad en la identificación de las desigualdades que actúan sobre las personas con diversidad funcional visual, en tanto permite comprender a través de las diferentes relaciones que se establecen entre las mismas, cuál es el espacio social que ocupan, cuáles son sus posibilidades de reacción y si los recursos de las administraciones potencian la igualdad o por el contrario perpetúan la desigualdad $d^{38,45,46}$.

La articulación de la interseccionalidad con las posturas de la salud pública alternativa, permite no solamente hacer un ejercicio comprensivo de la realidad de las personas con diversidad funcional visual, sino que también permite tener herramientas para poder superar las discriminaciones, ver íntegra la complejidad y especificidad de los asuntos de los derechos de las personas y establecer las condiciones necesarias para la garantía de sus derechos humanos, aspecto fundamental desde la mirada política y técnica de la salud pública.

\section{Referencias}

1. Palacios A. El modelo social de discapacidad: orígenes, caracterización y plasmación en la Convención Internacional sobre los Derechos de las Personas con Discapacidad. Primera Edición. Madrid: Grupo editorial CINCA; 2008. http:// riberdis.cedd.net/handle/11181/3624.

2. Morales Aguilera P, Vallés González B. Discapacidad y reconocimiento: reflexiones desde el prisma de Axel Honneth. Dilemata. 2013; 13: 189-208.

3. Hernandez Ríos MIH. El concepto de discapacidad: de la enfermedad al enfoque de derechos. Rev CES Derecho. 2015; 6: 46-59.

4. Romañach J, Palacios A. El modelo de la diversidad: una nueva visión de la bioética desde la perspectiva de las personas con diversidad funcional (discapacidad). Intersticios Rev Sociológica Pensam Crít. 2008; 2(2). http://www.intersticios.es/article/view/2712.

5. Universidad Nacional de Colombia. Salud pública y discapacidad. Primera Edicion. Bogotá; 2008. http://www.uneditorial.com/salud-publica-ydiscapacidad-salud-publica.html. 
6. Guisasola, L. Informe de la salud visual en Sudamérica 2008 - Cátedra UNESCO de salud visual y desarrollo - UPC. Universitat Politécnica de Catalunya; 2008. https://unescovision.upc. edu/es/materiales/materiales-de-la-catedra/ investigacion/savim/informe-de-la-salud-visual-ensudamerica-2008/view.

7. Cabrero JR, Rizzo AP. El modelo de la diversidad: una nueva visión de la bioética desde la perspectiva de las personas con diversidad funcional (discapacidad). Intersticios Rev Sociológica Pensam Crít. 2008; 2(2). http://www.intersticios.es/article/ view/2712.

8. Ale A. Convivencia paradójica de paradigmas de discapacidad. +E Rev Ext Universitaria. 2014; 4(4): 22-27. doi: https://doi.org/10.14409/extension. v1i4.4585.

9. Montoro J. Los ciegos en la historia. Madrid; 1992.

10. Lizama VV. Los modelos de la discapacidad: un recorrido histórico. Rev Empresa Humanismo. 2012; 15(1): 115-136.

11. Granda E. A qué llamamos salud colectiva, hoy. Rev Cuba Salud Pública. 2004; 30(2).

12. Céspedes GM. La nueva cultura de la discapacidad y los modelos de rehabilitación. Aquichán. 2005; 5(1): 108-113.

13. Villanueva López C, Eusebio Leyba J, Peralta Sánchez V. Las clasificaciones de la enfermedad y la discapacidad de la OMS. 2005; 27(5): 274-283. doi: https://doi.org/10.1016/S02115638(05)73450-7.

14. Van Dijik K, Kishiki E, Courtright P. Atención de la baja visión en África: enfoques prácticos para los servicios clínicos y la participación y planificación educativa. 2014. https:/www.iapb. org/wp-content/uploads/ESPANOL_FINAL.pdf.

15. Rojas LA. Psicología y discapacidad: un encuentro desde el paradigma social. Rev Costarric Psicol. 2013; 32(1): 63-74.

16. World Health Organization, ed. Clasificación estadística internacional de enfermedades y problemas relacionados con la salud. Décima revisión. [10a rev.]. Washington, D.C: OPS, Oficina Sanitaria Panamericana, Oficina Regional de la Organización Mundial de la Salud; 1995.

17. World Health Organization. International Classification of Functioning, Disability and Health (ICF). 2001. https://www.who.int/ classifications/icf/en/.

18. Franco Agudelo S. Teoría y práctica de la salud pública. Rev Fac Nal Salud Pública. 1995; 12(2): 63-76.
19. Minto H, Awan H. Establishing low vision services at secondary level. Community Eye Health. 2004; 17(49): 5.

20. Verdugo Alonso MA. La concepción de discapacidad en los modelos sociales (SID). Inv Innov Cambio. 2003; 235-247.

21. Ferrante C. Usos, posibilidades y dificultades del modelo social de la discapacidad. Rev Inclusiones. 2014; 1(3): 31-55.

22. Padilla-Muñoz A. Discapacidad: contexto, concepto y modelos. Int Law Rev Colomb Derecho Int. 2010; 16: 381-414. http://www.redalyc.org/resumen. oa? id $=82420041012$.

23. Fernández-López JA, Fernández-Fidalgo M, Geoffrey R, Stucki G, Cieza A. Funcionamiento y discapacidad: la Clasificación Internacional del Funcionamiento (CIF). Rev Esp Salud Pública. 2009; 83: 775-783. doi: https://doi.org/10.1590/ S1135-57272009000600002.

24. Seoane JA. ¿Qué es una persona con discapacidad? Ágora Pap Filosofía. 2011; 30(1): 143-161. https:// minerva.usc.es/xmlui/handle/10347/7386.

25. Vanegas García JH, Gil Obando LM. La discapacidad, una mirada desde la teoria de sistemas y el modelo biopsicosocial. Hacia Promoc Salud. 2007; 12: 51-61.

26. Jiménez Buñuales MT, Paulino González D, Moreno JM. La clasificación internacional del funcionamiento de la discapacidad y de la salud (CIF) 2001. Rev Esp Salud Pública. 2002; 76(4): 271-279.

27. Coco Martín MB, Herrera Medina J, de Lazaro Yagüe JA, Cuadrado Asencio R. Manual de baja visión y rehabilitación visual. Ed Médica Panamericana; 2015.

28. Rodríguez Díaz S, Ferreira MA. Diversidad funcional: Sobre lo normal y lo patológico en torno a la condición social de la dis-capacidad. Cuad Relac Laborales. 2010; 28(1): 151-172.

29. Canimas Brugué J. ¿Discapacidad o diversidad funcional? Siglo Cero Rev Esp Sobre Discapac Intelect. 2015; 46(2): 79. doi: https://doi. org/10.14201/scero20154627997.

30. Chiang PP, O'Connor PM, Le Mesurier RT, Keeffe JE. A global survey of low vision service provision. Ophthalmic Epidemiol. 2011; 18(3): 109-121. doi: https://doi.org/10.3109/09286586. 2011.560745.

31. Lam N, Leat SJ. Barriers to accessing low-vision care: the patient's perspective. Can J Ophthalmol. 2013; 48(6): 458-462. doi: https://doi.org/10.1016/j. jcjo.2013.02.014. 
32. Valencia M. Aportes de los nuevos enfoques para la conformación de la salud pública alternativa. Rev Fac Nac Salud Pública. 2011; 29(1): 85-93.

33. Organización Mundial de la Salud. Ceguera y discapacidad visual. World Health Organization. http://www.who.int/es/news-room/fact-sheets/ detail/blindness-and-visual-impairment.

34. Victoria Maldonado JA. El modelo social de la discapacidad: una cuestión de derechos humanos. Bol Mex Derecho Comp. 2013; 46(138): 1093-1109.

35. Oviedo Cáceres MP, Hernández M, Ruíz M. Baja visión en Colombia: una situación invisible para el país. Rev Fac Nac Salud Pública. 2015; 33(1): 22-30.

36. Granda, Edmundo. La salud pública y las metáforas sobre la vida. Rev Fac Nac Salud Pública. 2000; 18(2): 83-100.

37. La Barbera, María Caterina. Interseccionalidad. Eunomía Rev En Cult Leg. 2017; 12: 191-198. doi: https://doi.org/10.20318/eunomia.2017.3651.

38. Viveros Vigoya M. La interseccionalidad: una aproximación situada a la dominación. Debate Fem. 2016; 52: 1-17. doi: https://doi.org/10.1016/j. df.2016.09.005.

39. La Barbera MC. Interseccionalidad, un "concepto viajero": orígenes, desarrollo e implementación en la Unión Europea. Interdisciplina. 2016; 4(8): 105-122. doi: http://dx.doi.org/10.22201/ ceiich.24485705e.2016.8.54971.

40. Mora-Ríos J, Bautista N. Estigma estructural, género e interseccionalidad. Implicaciones en la atención a la salud mental. Salud Ment. 2014; 37(4): 302. doi: https://doi.org/10.17711/SM.0185-3325.2014.035.

41. González RG. Interseccionalidad y salud pública en el ámbito de las relaciones erótico-afectivas entre adolescentes en Colombia. Athenea Digit Rev Pensam E Investig Soc. 2018; 18(3): 2070. doi:10.5565/rev/athenea.2070.

42. Palacios A, Romañach J. El modelo de la diversidad: la bioética y los derechos humanos como herramientas para alcanzar la plena dignidad en la diversidad funcional. Diversitas; 2006. https:// e-archivo.uc3m.es/handle/10016/9899.

43. Crenshaw K. Demarginalizing the intersection of race and sex: a black feminist critique of antidiscrimination doctrine, feminist theory and antiracist politics. Univ Chic Leg Forum. 2015; 1989(1). https://chicagounbound.uchicago.edu/uclf/ vol1989/iss $1 / 8$.

44. Symington S. Interseccionalidad: una herramienta para la justicia de género y la justicia económica. Derechos Las Mujeres Cambio Económico. 2004; 9.
45. Molina CE. ¿Qué es eso de la interseccionalidad? Aproximación al tratamiento de la diversidad desde la perspectiva de género en España. Investig Fem. 2013; 3(0): 203-222. doi: https://doi.org/10.5209/ rev_INFE.2012.v3.41146.

46. Gómez Bernal V. Análisis de la discapacidad desde una mirada crítica: Las aportaciones de las teorías feministas. Estud Pedagógicos Valdivia. 2014; 40(2): 391-407. doi: https://doi.org/10.4067/S071807052014000300023. 